\title{
Tamponamiento cardiaco secundario a pericarditis purulenta: reporte de un caso
}

\author{
Alejandro J. Moya-Álvarez ${ }^{1 *}$, David J. Hernández-Solano² y Rebeca Jiménez-Murillo ${ }^{3}$ \\ ${ }^{1}$ Medicina de Emergencias, Hospital Dr. Rafael Ángel Calderón Guardia; ${ }^{2}$ Área de salud de Oreamuno, Clínica Dr. Ricardo Jiménez Núñez; \\ ${ }^{3}$ Medicina de Emergencias, Programa Nacional de Residencia en Medicina de Emergencias, Sede Hospital Dr. Rafael Ángel Calderón Guardia. \\ Caja Costarricense del Seguro Social, San José, Costa Rica
}

\begin{abstract}
Resumen
Actualmente, la pericarditis purulenta representa un completo reto a la hora de realizar un adecuado diagnóstico y abordaje. La primera razón de ello es debido a su rareza epidemiológica, la cual exige tenerla siempre entre los diagnósticos diferenciales de un paciente con clínica de pericarditis. Otra razón es la etiológica, debido a los cambios en el aspecto bacteriológico responsable de dicha patología, ya que desde su descubrimiento y por el uso cada vez mayor de fármacos antibióticos han ido en aumento los diagnósticos por gérmenes anaerobios, gramnegativos y hongos, frente a los clásicos aerobios grampositivos que eran en su mayoría el agente causal en otras épocas. El otro aspecto que debe destacarse es que aun en nuestros tiempos, con todo el avance que ha tenido la medicina, persiste la controversia en cuanto al mejor abordaje para estos pacientes. Por tanto, desarrollamos una propuesta de un algoritmo diagnóstico y de manejo para hacer más eficiente el abordaje de esta patología e incidir de manera favorable en el pronóstico. Presentamos el caso de una pericarditis purulenta en un paciente masculino en el cual la ecografía al pie de la cama acortó los tiempos de diagnóstico y manejo, lo cual impactó de una manera muy favorable en el pronóstico.
\end{abstract}

Palabras clave: Pericarditis purulenta. Taponamiento cardiaco. Derrame pericárdico.

\section{Cardiac tamponade due to purulent pericarditis: A case report}

\section{Abstract}

Purulent pericarditis now a days, represents a real challenge in the clinical practice. One of the reasons is due to its epidemiological aspects which obligates it to be always present as a differential diagnosis of patients with pericarditis. Another important reason is its etiology because of changes in bacteriological aspects. The continued use of more antibiotics has given rise to anaerobic, gram negative and fungal infections in favor of the classical aerobic gram-positive infections that were the typical causal agents before. The other aspect that must be taken into consideration is that even with our technological advantages there is still debate on how to best approach our patients. For that reasons we developed a propose of diagnostic and management algorithm to simplify the approach of this disease and to have better outcomes. We present the case of a purulent pericarditis in a male, whose bedside echocardiography shortened the time of diagnostic, impacting time to treatment which had a positive impact in his prognosis.

Key words: Purulent pericarditis. Cardiac tamponade. Pericardial efussion.

\section{Correspondencia}

*Alejandro J. Moya-Álvarez

E-mail: amoya@resusimtation.com
Disponible en internet: 13-09-2021 Rev Educ Investig Emer. 2021;3(3):174-181 www.medicinadeemergencias.com (6) 2021 Sociedad Mexicana de Medicina de Emergencias, AC. Publicado por Permanyer México SA de CV. Este es un artículo open access bajo la licencia CC BY-NC-ND (http://creativecommons.org/licenses/by-nc-nd/4.0/). 


\section{Introducción}

La pericarditis purulenta (PP) se define como la ocupación del saco pericárdico por fluido purulento. Esta entidad representa una patología de curso letal si no se aborda con prontitud; hoy en día su mortalidad reportada en la literatura oscila entre el 2 y el $20 \%{ }^{1}$. La PP es producida clásicamente por gérmenes aerobios grampositivos, sin embargo, en publicaciones más recientes se reconoce que la incidencia de etiología anaerobia, gramnegativa y fúngica parece ir en aumento.

La etiología neumocócica es hoy en día excepcional, lo que hace que sea tan particular el caso de esta revisión, debido principalmente a la cada vez mayor disponibilidad de mejores y diversas clases de antibióticos; no obstante, la PP por este germen prácticamente siempre se acompaña de una infección pleuropulmonar evidente. Por otra parte, en la actualidad se reconoce a Staphylococcus aureus como el agente más frecuente ${ }^{2,3}$.

\section{Presentación del caso}

Se presenta el caso de un hombre de 45 años, vecino de la capital, San José, Costa Rica. Con antecedente de tabaquismo de uno y medio paquetes por día durante 12 años. No presentaba otros antecedentes de importancia para el padecimiento actual. Negó tener enfermedades cardiovasculares previas.

El paciente fue traído por el personal prehospitalario con historia de una semana de evolución de disnea progresiva y dolor torácico de carácter punzante con patrón in crescendo; dicho cuadro había estado precedido por un episodio de tos seca y de autorresolución espontánea 15 días previos al inicio de la disnea.

Además, asociaba astenia, adinamia, dolor abdominal difuso y edema generalizado. Al ingreso a nuestro hospital se encontraba febril $\left(38.3^{\circ} \mathrm{C}\right)$, presentando una presión arterial de 90/60 $\mathrm{mmHg}$, frecuencia cardiaca de $112 \mathrm{lpm}$ y frecuencia respiratoria de $22 \mathrm{rpm}$. Consciente, orientado, sin lograr tolerar el decúbito dorsal, con ingurgitación yugular grado II. Los ruidos cardiacos eran rítmicos hipofonéticos, pero no se auscultaban soplos ni frote pericárdico. Los pulmones presentaban disminución del murmullo bilateral de predominio izquierdo y algunos crepitantes. Además, abdomen blando y depresible, sin visceromegalias, sin embargo, presentaba discreto dolor a la palpación, difuso, sin encontrar datos sugestivos de irritación peritoneal y peristaltismo normal. Presentaba edema de

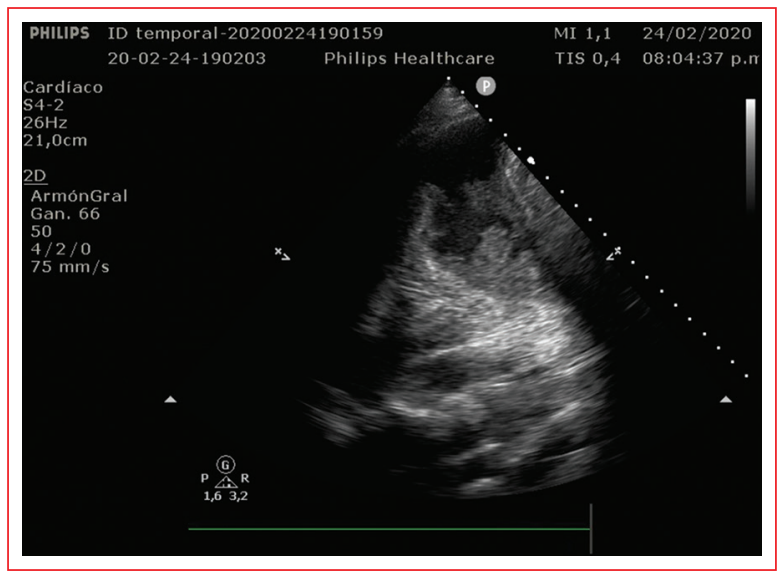

Figura 1. Ecografía a pie de la cama del paciente que muestra derrame pleural con tabicaciones en hemitórax izquierdo.

extremidades hasta tercio medio, no doloroso. Pulsos periféricos normales.

Los laboratorios realizados al ingreso reportaron hemoglobina en $10.5 \mathrm{~g} / \mathrm{dl}$, un hematocrito del $32 \%$, plaquetas 262,000, un conteo total de leucocitos en 18,820 con predominio de segmentados en un $86 \%$, una proteína $C$ reactiva que se encontraba en $16.21 \mathrm{mg} / \mathrm{dl}$ y una albúmina en $2.6 \mathrm{mg} / \mathrm{dl}$. Finalmente, su función renal, hepática y pruebas de coagulación no mostraban alteraciones.

Dada su inestabilidad al ingreso se realizó aproximación con ecografía al pie de la cama del paciente, documentando la presencia de derrame pleural bilateral (Fig. 1) y derrame pericárdico con signos de taponamiento cardiaco (Fig. 2), por lo que se decide realizar pericardiocentesis diagnóstica y terapéutica, logrando extraer $400 \mathrm{cc}$ de material purulento (Fig. 3). Debido al hallazgo previo se coloca sonda endopleural izquierda, obteniendo $1,700 \mathrm{cc}$ de material de similares características (Fig. 4).

Un electrocardiograma realizado posteriormente mostraba únicamente taquicardia sinusal, con un eje cardiaco normal y bajo voltaje electrocardiográfico. No se encontraron cambios agudos sugerentes de isquemia miocárdica.

Se inició cobertura antibiótica empírica intravenosa con cefotaxima, vancomicina y metronidazol con evolución satisfactoria del paciente durante su estancia en el servicio de emergencias.

El cultivo del líquido drenado del saco pericárdico, así como el del drenaje pleural, fueron positivos por infección secundaria a Streptococcus pneumoniae. El 


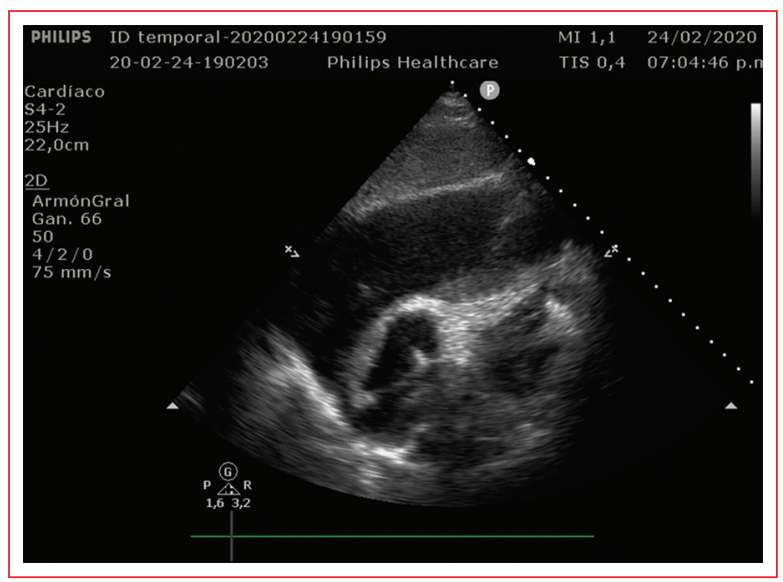

Figura 2. Ecografía a pie de la cama del paciente que muestra derrame pericárdico importante.

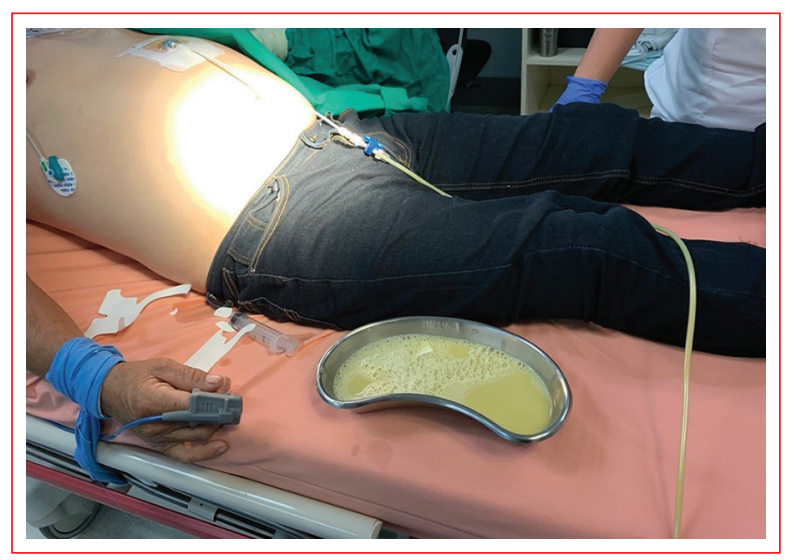

Figura 3. Pericardiocentesis guiada por ecografía que muestra un drenaje purulento desde el saco pericárdico.

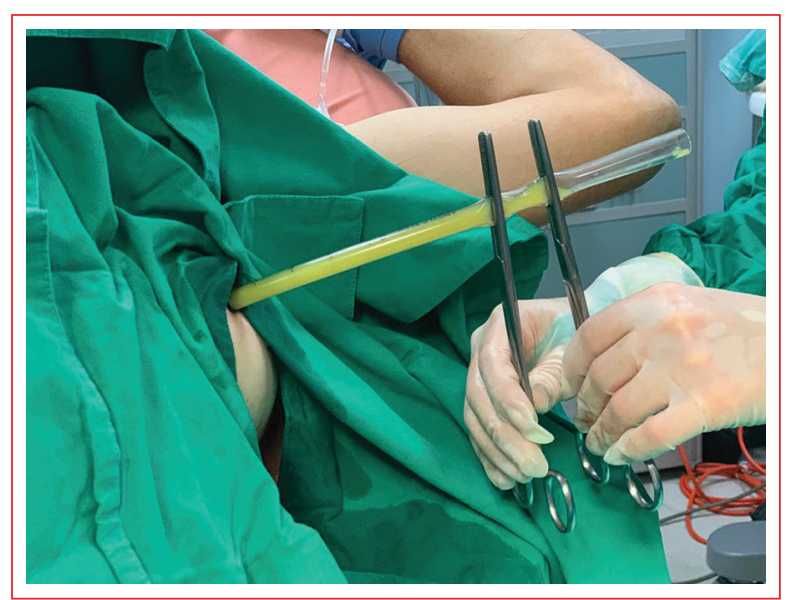

Figura 4. Colocación de tubo de drenaje torácico que muestra salida de material de similares características al obtenido del saco pericárdico. paciente fue ingresado al servicio de cirugía de tórax para su seguimiento y evaluación definitiva.

\section{Discusión y revisión de la literatura}

La PP no es un diagnóstico frecuente, mucha de la literatura concuerda en que representa aproximadamente $<1-2 \%$ de los casos ${ }^{4,5}$; no obstante, es un diagnóstico que no debe pasarse por alto, ya que en registros históricos, sobre todo en la era preantibiótica, la ausencia de un diagnóstico oportuno se acompaña de una mortalidad cercana al $100 \%$.

Aunado a esto, en 9 de cada 10 casos de pericarditis aguda se reconocen entre las principales etiologías la viral y desconocida (idiopática) ${ }^{7}$. En niños, la pericarditis aguda es menos frecuente que en adultos, siendo la causa infecciosa principal los agentes virales, como por ejemplo: adenovirus, influenza o coxsackie. Diversas bacterias han sido reportadas como causantes de PP, incluyendo $S$. aureus, Neisseria meningitidis, Streptococcus viridans, Haemophilus influenzae, Streptococcus pyogenes y, menos frecuentemente, los bacilos entéricos gramnegativos y anaerobios.

Existen diversas pautas de diagnóstico y abordaje terapéutico descritas; sin embargo, debido a los pocos casos reportados, la técnica idónea para tratar a dichos pacientes continúa siendo hoy en día un motivo de controversia, según concuerdan todas las literaturas revisadas, no obstante, la rareza de esta patología es algo que nos exige tenerla siempre en mente para evitar un retardo en el diagnóstico y pronóstico; lo cual podría resultar en un desenlace fatal.

Es importante destacar la relevancia que esto conlleva, ya que Ferreira Dos Santos, et al. mencionan que en un estudio retrospectivo realizado en un hospital español, con una población de 593,601 pacientes entre los años 1972-1991, se encontraron 33 casos de PP, de los cuales solo el $19 \%$ se diagnosticaron in vivo. Básicamente, esto debido a que nunca se consideró dicha patología entre los diagnósticos diferenciales ${ }^{5}$.

\section{Fisiopatología}

El pericardio es una capa serosa que envuelve al corazón y se encuentra formada por dos hojas, la visceral y la parietal. El pericardio parietal tiene una capa externa fibrosa compuesta, a su vez, de múltiples capas de fibras colágenas intercaladas con fibras elásticas y una serosa con microvellosidades especializadas en la secreción de líquido pericárdico. 
A la inflamación del pericardio se le denomina pericarditis. Esta inflamación puede estar acompañada de derrame en escasa cantidad o en un mayor volumen que llegue incluso a causar taponamiento cardiaco. Se considera como aguda si tiene una duración igual o menor a doce semanas ${ }^{8}$.

La presencia de líquido en el espacio pericárdico superior a lo normal ( $1 \mathrm{ml} / \mathrm{kg}$ o hasta $15 \mathrm{ml}$ de líquido) acompaña casi constantemente a la pericarditis clínica, de modo que la ausencia de derrame a ciertas edades o por distintas causas o curso evolutivo de la enfermedad hace en ocasiones difícil sustentar el diagnóstico de pericarditis ${ }^{3,8}$.

Existen cinco mecanismos que explican la invasión del espacio pericárdico en la PP: a) propagación desde un sitio intratorácico, por ejemplo, un foco neumónico; b) diseminación hematógena; c) lesión miocárdica; d) lesión perforante o cirugía, sobre todo torácica, y e) propagación desde algún sitio subdiafragmático.

La infección neumocócica más frecuentemente se ha asociado a diseminación desde un sitio intratorácico, mientras que $S$. aureus está más frecuentemente implicado en mecanismos de diseminación hematógena ${ }^{5,9}$.

La patogenia de síntomas y signos está determinada por el aumento de la presión intrapericárdica, el cual depende no solo de la cantidad absoluta de líquido, sino también de la rapidez de su instalación y de las características del saco pericárdico. Como es de esperar, el aumento rápido en la cantidad de líquido acumulado a nivel de esta serosa es peor tolerado hemodinámicamente hablando.

El taponamiento cardiaco se comporta en estos casos como el resultante de un inadecuado llenado diastólico, y presenta un continuum en cuanto a sucesión de gravedad, que puede ir desde aumentos ligeros de la presión intrapericárdica sin repercusión clínicamente evidente, hasta un cuadro grave de hipoperfusión y muerte ${ }^{3}$.

La PP comparte diversas características con el empiema. En el caso de este último, la diseminación de focos neumónicos contiguos genera una disfunción de la barrera mesotelial, desembocando en un derrame pleural con características exudativas. Otro hallazgo consecutivo es la invasión bacteriana, la cual se comporta como factor quimiotáctico para leucocitos al espacio pleural. Consecutivamente estos leucocitos activados estimulan la cascada de la coagulación, resultando en la formación de fibrina ${ }^{6,10}$.

Estos depósitos fibrinoides son responsables de la adhesión pleural, espesamiento y tabicaciones en el material acumulado, dificultando así el drenaje exitoso de dicho fluido y la penetración antibiótica hacia estos tejidos. Finalmente, mientras este proceso se prolonga en el tiempo y asociado a la inflamación resultante derivará en un desenlace inevitable hacia un proceso fibrótico debido a la secreción de colágeno ${ }^{10}$.

Diversos modelos experimentales describen un proceso fisiopatológico similar en la PP, trascendiendo desde una inflamación pericárdica hasta adhesión y fibrosis en dicha capa. Por todos estos hallazgos, diversos estudios han mostrado cómo la formación de fibrina es la piedra angular en la patogénesis tanto de la PP persistente como de la pericarditis constrictiva.

\section{Diagnóstico}

Como ya se indicó anteriormente, nueve de cada 10 casos de pericarditis aguda responden a causas infecciosas (sobre todo de origen viral) e idiopáticas. A esto puede añadirse, sin embargo, que la aplicación de un proceso diagnóstico sistemático disminuye el porcentaje de pericarditis consideradas como idiopáticas, lo que pone de manifiesto un número mayor de casos más graves que puedan beneficiarse de un tratamiento específico ${ }^{11}$ y una de estas es la PP que se presenta en este caso.

Hoy en día el diagnóstico se basa en: a) dolor retroesternal; b) la auscultación del frote pericárdico; c) cambios electrocardiográficos sugestivos; d) ecocardiograma, y e) datos clínicos de taponamiento cardiaco. Sin embargo, Suberviola, et al. mencionan que el diagnóstico se sustenta sobre dos pilares fundamentales, la clínica y el ecocardiograma ${ }^{8,9}$.

\section{Manifestaciones clínicas}

El diagnóstico clínico es complicado, ya que en los niños prevalecen los síntomas inespecíficos, así el frecuente dolor precordial, el cual es un síntoma predominante en adultos, puede pasar inadvertido en niños pequeños o manifestarse solamente por incomodidad e irritabilidad.

Los síntomas suelen ser de aparición aguda e inespecíficos, predominando la fiebre, taquicardia, taquipnea y ataque al estado general sobre los síntomas típicos de la pericarditis per se. Por lo anterior debe comprenderse y tomarse en cuenta que un paciente séptico o con alguna otra comorbilidad o factor de riesgo que pueda explicar el derrame pericárdico conllevan que el reconocimiento temprano de la PP sea 
más complejo de identificar que en otros tipos de pericarditis ${ }^{3-5}$.

Dentro de los signos al examen físico, debe buscarse la caída en la presión de pulso, presencia de pulso paradójico (disminución mayor a $10 \mathrm{mmHg}$ en la presión arterial sistólica durante la inspiración), tonos cardiacos apagados, frote pericárdico e ingurgitación yugular ${ }^{7,8}$.

\section{Laboratorio y gabinete}

El ecocardiograma es la técnica de exploración más útil para identificar la presencia y cuantificar el derrame pericárdico, así como su seguimiento. Esto, además, aporta datos de suma importancia para establecer el diagnóstico de taponamiento, principalmente colapso de la aurícula o del ventrículo derechos, así como variación transmitral y transcuspídea. Sin embargo, se debe hacer énfasis en que los signos ecocardiográficos más sensibles para el diagnóstico de taponamiento parecen ser el colapso telediastólico de la aurícula derecha y el colapso diastólico ventricular derecho $0^{1,3,9}$.

Otra de las ventajas que presenta la ecocardiografía es que, aparte de permitir una excelente aproximación diagnóstica, algunos autores mencionan que la sensibilidad del estudio transtorácico para detectar derrame pericárdico en manos de un buen operador se aproxima al $100 \%$; además, permite una guía visual para realizar una pericardiocentesis, ayudando a completar así un diagnóstico etiológico más fidedigno, y por otra parte ayuda a la terapéutica al generar una descompresión pericárdica y mejoría en la función hemodinámica, así como en el retorno venoso al corazón.

Más recientemente, otros autores han descrito la utilidad de la tomografía computarizada como método diagnóstico precoz ante la sospecha de existencia de derrame pericárdico. Se trata de un método no invasivo, al igual que la ecocardiografía, y permite, además, no solo cuantificar la magnitud del derrame, sino también realizar una aproximación diagnóstica a la etiología de este ${ }^{10}$.

En cuanto a la electrocardiografía, Donoso, et al. describen que los cambios típicos encontrados en la pericarditis de otras etiologías son más raros de encontrar en la PP, como la elevación difusa del segmento ST, la depresión de voltaje y la alternancia eléctrica. Estos dos últimos suelen detectarse más comúnmente ante un derrame extenso.

El único dato relevante en esta patología en el que conviene destacar la importancia de la radiografía de tórax es que a pesar de considerarse poco confiable, ya que se requieren aproximadamente más de $200 \mathrm{ml}$ de fluido acumulado para poder sospechar un derrame pericárdico, es que, en proyección lateral la presencia de línea grasa pericárdica, aunque infrecuente, es un hallazgo altamente sugestivo de efusión pericárdica masiva ${ }^{3}$.

En cuanto a la elevación en la concentración de troponinas cardiacas, se ha visto que no se correlaciona con un peor pronóstico, sino que más bien responde a la inflamación epicárdica y pericárdica, más que a una necrosis de los miocitos. Por otra parte, sí debe rescatarse que la elevación persistente en el tiempo (más de dos semanas) sugiere la coexistencia de miocarditis, la cual ensombrece el pronóstico $0^{3,10}$.

Debe tenerse en cuenta que la última edición de las guías americanas del corazón, así como distintos autores $^{3-5,10}$, mencionan que la sola sospecha de PP es indicación de realizar una pericardiocentesis urgente; la cual resulta no solamente terapéutica por la extracción del fluido patológico y la descompresión de la cavidad pericárdica, sino que también resulta diagnóstica.

Lo anterior toma mayor importancia cuando el fluido extraído mediante esta técnica resulta francamente purulento4; no obstante, distinguir a simple vista un líquido purulento de uno turbio es sumamente complicado, además de que puede conllevar serios errores diagnósticos. Debe tenerse en cuenta que un fluido puramente turbio en una pericardiocentesis obliga a descartar en primera instancia infección (PP) y malignidad, visualmente resulta interesante hacer notar que la literatura reporta este tipo de fluido también en pericarditis tuberculosa, sin embargo, más que puramente turbio lo reportan como un líquido turbio con estrías sanguinolentas ${ }^{12}$.

Conviene destacar en este apartado distintos valores que ayudan a orientar hacia un adecuado diagnóstico: cociente glucosa en líquido pericárdico: glucosa sérica, cuyo valor normal es cercano a $1^{12}$, además del conteo de glóbulos blancos y porcentaje de neutrófilos encontrados en el fluido pericárdico. Una razón de glucosa baja (cercana a 0.3 ), conteo de leucocitos elevados con un porcentaje alto de neutrófilos (> 2.8 leucocitos $/ \mu$, $92 \%$ neutrófilos) ayuda a diferenciar el líquido purulento de uno tuberculoso (razón de glucosa cercana a 0.7, 1.7 leucocitos/ $\mu$ l, $50 \%$ neutrófilos).

Finalmente, una razón de glucosa cercana a 0.8 (3.3 leucocitos/ $\mu$ l, $55 \%$ neutrófilos) orientará más hacia malignidad. El clínico, aparte de orientar el diagnóstico con estos valores anteriormente citados, debe enviar una muestra del líquido extraído para realización de frotis y cultivo por bacterias, hongos y tuberculosis, así como otras muestras de acuerdo con la presentación clínica de cada paciente según corresponda ${ }^{4,12}$. 


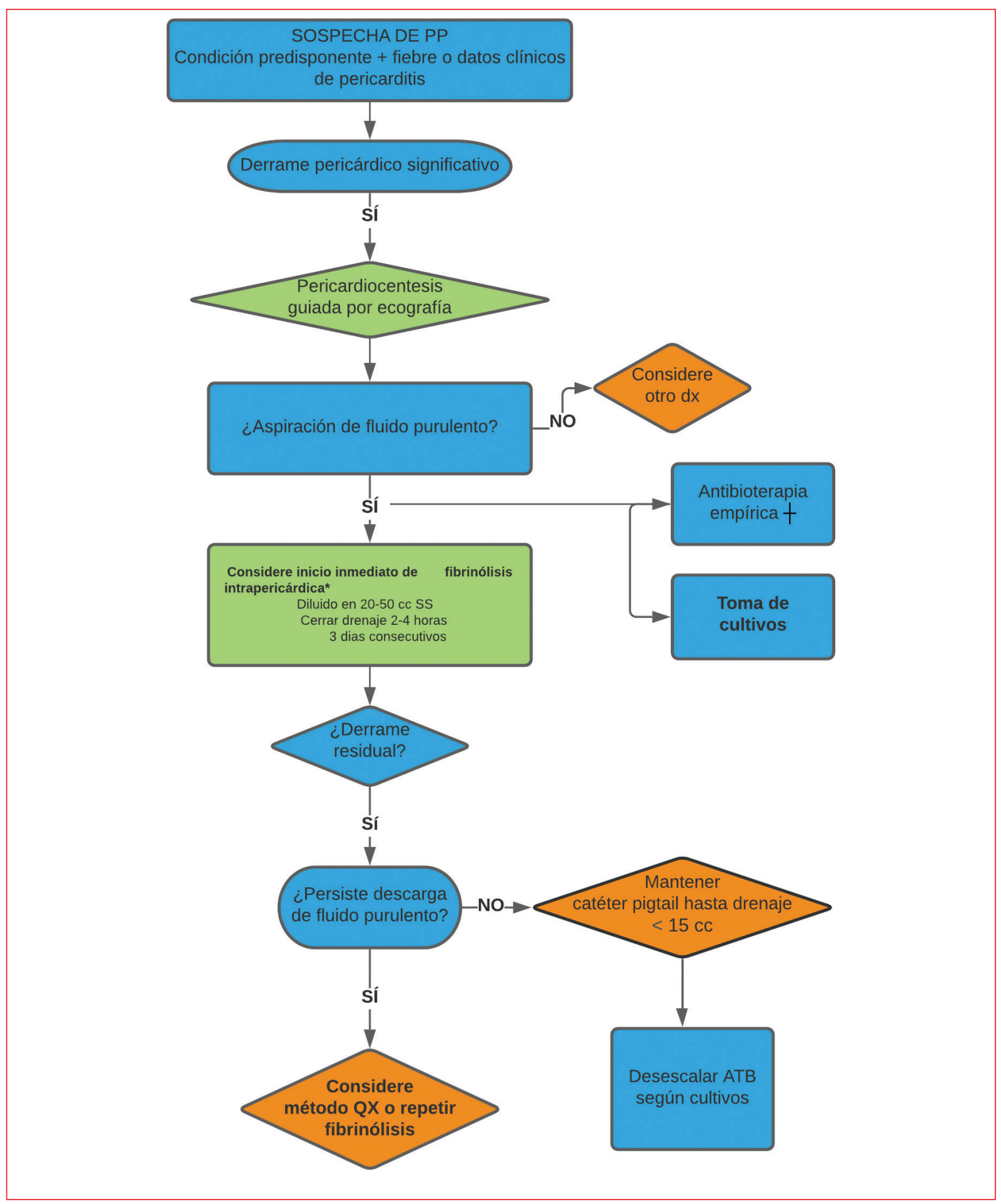

Figura 5. Algoritmo propuesto para el manejo de la pericarditis purulenta. Nótese que el tratamiento antibiótico y la toma de cultivos se colocan en un mismo nivel, ya que debe de ser un procedimiento que se realice de manera concomitante; preferentemente con la toma de cultivos inicialmente si la situación clínica del paciente así lo permite.

*Esquema de posología tomado de The British Thoracic Society guidelines. Dosis: estreptocinasa 250,000 UI/día; urocinasa 200,000 Ul/día; tenecteplasa $10 \mathrm{mg} /$ día $^{10}$.

${ }^{\dagger}$ ATB empírica: ver apartado de tratamiento en el texto y la tabla 1.

PP: pericarditis purulenta; Dx: diagnóstico; SS: solución salina (0.9\%); 0x: método quirúrgico; ATB: antibiótico. 
Tabla 1. Régimen recomendado y dosis de tratamiento en pericarditis purulenta

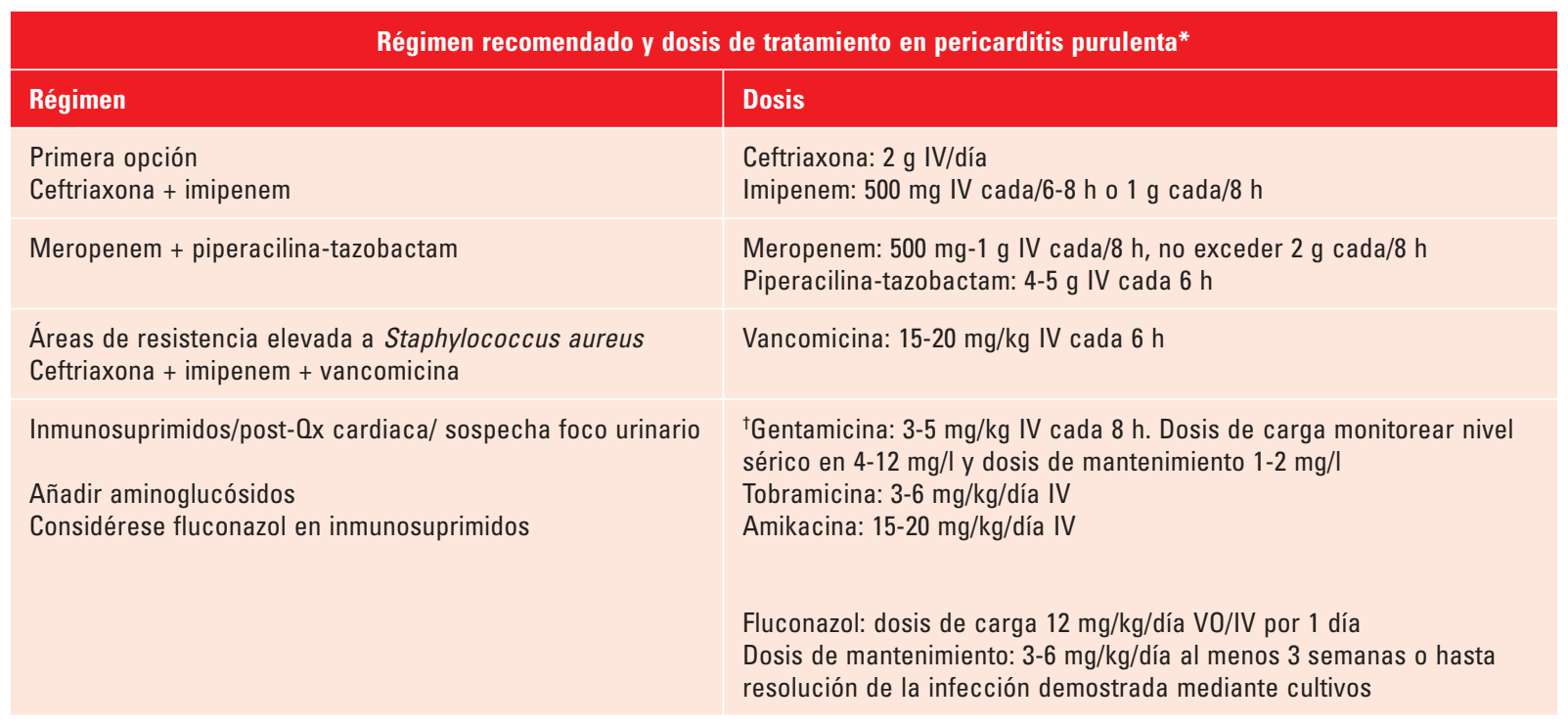

*La duración del tratamiento debe guiarse con la mejoría clínica y ecocardiográfica. Léase en el apartado de tratamiento que la mayoría de los autores recomiendan tratar por al menos 3-4 semanas.

†En la recomendación de los aminoglucósidos se indican los 3 que según indica la literatura se utilizan con más frecuencia.

IV: intravenoso; VO: vía oral; 0x: cirugía.

Adaptada de Medscape, $2020^{14}$.

\section{Tratamiento}

Hasta la década de 1940 la mortalidad de la PP era cercana al $100 \%$ en casos no tratados y/o mal diagnosticados. Posterior a esta época el advenimiento de la pericardiostomía redujo dichas cifras a un $50 \%$ aproximadamente e incluso a un $30 \%$ cuando se le asociaba a un régimen de antibioterapia adecuada ${ }^{10}$.

Además, distintos autores dejan claramente demostrado cómo la eliminación del fluido infectado dentro de la bolsa pericárdica resulta una piedra angular en el tratamiento de la PP; no obstante, el método más fiable para dicha extracción continúa siendo hasta la fecha un motivo de controversia.

La pericardiocentesis permite realizar el diagnóstico bacteriológico mediante el análisis del líquido y descomprimir a los ventrículos en situaciones de urgencia. Es recomendable dejar un catéter pigtail con drenaje continuo para disminuir el riesgo de recurrencias. En algunas ocasiones, la aspiración completa del líquido purulento mediante esta técnica resulta casi imposible, debido a que el derrame podría estar compartimentado (tabicado); así mismo, el pigtail puede obstruirse debido a la alta viscosidad del material purulento, por tanto, algunos pacientes que sobreviven tras la realización de esta maniobra pueden desarrollar con el tiempo pericarditis constrictiva debido al intenso componente inflamatorio asociado ${ }^{13}$.
Ante este tipo de eventos, la pericardiostomía subxifoidea habitualmente permite un mejor drenaje, ya que hace posible liberar manualmente las adherencias; además de ser el método recomendado por las Guías de la Sociedad Europea de Cardiología'.

Se han empleado distintas técnicas, entre las habituales: pericardiostomía subxifoidea, ventana pericárdica (a pleura o a peritoneo) y la pericardiectomía. La manera idónea no ha sido satisfactoriamente esclarecida empero, la pericardiectomía es necesaria en el grupo de pacientes que desarrollan constricción pericárdica sostenida o taponamiento recurrente pese al empleo de maniobras menos agresivas. Algunos expertos defienden la idea de un drenaje quirúrgico agresivo si dos pericardiocentesis evacuadoras resultan fallidas ${ }^{2}$.

La fibrinólisis intrapericárdica es recomendada con un buen grado de evidencia según guías internacionales, así como diversos expertos en el tema. El primer caso de una fibrinólisis intrapericárdica exitosa data del año de 1951, donde olvidada por aproximadamente 30 años fue redescubierta por Benneth, et al. en 1984, mostrando un drenaje más eficaz de la cavidad pericárdica que cuando se realizaba solo mediante pericardiocentesis evacuadora, independientemente del método utilizado. Debido a los múltiples protocolos y medicamentos utilizados para realizar dicha técnica y que aún es un tópico en el cual se requieren más estudios para 
conocer de un modo más certero todos los datos a favor y en contra de su práctica, no se recomienda que se realice de una manera sistemática ${ }^{4,10}$.

Las dosis y la manera de utilizarla se describen en la figura 1; sin embargo, debe hacerse hincapié en que los distintos autores describen en ocasiones diversas pautas terapéuticas y no parece que se haya estandarizado dicho apartado. No obstante, en esta revisión se tomará en cuenta la posología recomendada por The British Thoracic Society, ya que se considera según todo lo revisado de manera exhaustiva que esta asociación es quien brinda las dosis de una manera más uniforme y sus resultados han sido satisfactorios.

La duración del tratamiento es empírica, pero habitualmente se recomienda prolongar por al menos 3-4 semanas. El tratamiento antibiótico inicial debe cubrir $S$. aureus y bacilos gramnegativos, recomendándose una combinación de un betalactámico resistente a penicilinasa con una cefalosporina de $3 .^{a}$ generación, considerando el uso de vancomicina en áreas de elevada tasa resistencia estafilocócica. Se recomienda además la asociación de un aminoglucósido en casos de: inmunosupresión, postoperatorio de cirugía cardiaca o cuando el foco primario es genitourinario. La antibioterapia intrapericárdica no parece tener especial indicación, dada la excelente penetración de los antimicrobianos en la cavidad pericárdica ${ }^{2,3,9}$. En la tabla 1 se muestra un esquema de tratamiento empírico dirigido a los principales agentes etiológicos de la PP.

Con base en toda la literatura revisada se propone un algoritmo diagnóstico y de tratamiento en la figura 5 , ya que al ser una patología con una mortalidad tan elevada, debe existir una manera más rápida, certera y eficaz de tratar a estos pacientes. Nótese además como en este flujograma destacan mucho los métodos invasivos, ya que, a pesar de no existir un consenso en cuanto al manejo de esta patología al ser una entidad tan infrecuente, nuestra experiencia apoya la opinión de otros autores, partidarios de un manejo más agresivo frente a un tratamiento conservador.

\section{Financiamiento}

La presente investigación no ha recibido ninguna beca específica de agencias de los sectores públicos, comercial o sin ánimo de lucro.

\section{Conflicto de intereses}

Los autores declaran no tener conflicto de intereses.

\section{Responsabilidades éticas}

Protección de personas y animales. Los autores declaran que para esta investigación no se han realizado experimentos en seres humanos ni en animales.

Confidencialidad de los datos. Los autores declaran que han seguido los protocolos de su centro de trabajo sobre la publicación de datos de pacientes.

Derecho a la privacidad y consentimiento informado. Los autores han obtenido el consentimiento informado de los pacientes y/o sujetos referidos en el artículo.

\section{Bibliografía}

1. García Martín LA, Campo Linares R, Rayo Gutiérrez M. Pericarditis purulenta: diagnóstico ecográfico precoz en el servicio de urgencias. Emergencias. 2008;20(2):135-8.

2. Albalá Martínez N, Moneo González A, Waez Tatari B, Argüelles Baquero A, Ferrero Zorita J, Martín Benítez JC. Pericarditis neumocócica: presentación de un caso y revisión de la literatura. Med Intensiva. 2005;29(5):308-12.

3. Donoso A, Díaz F, Bertrán K. Pericarditis purulenta primaria por Streptococcus pneumoniae, a propósito de un caso clínico. Rev Chil Pediatr. 2008;79(6):623-8.

4. Adler Y, Charron P, Imazio M, Badano L, Barón-Esquivias G, Bogaert J, et al. 2015 ESC Guidelines for the diagnosis and management of pericardial diseases: The Task Force for the Diagnosis and Management of Pericardial Diseases of the European Society of Cardiology (ESC) Endorsed by: The European Association for Cardio-Thoracic Surgery (EACTS). Eur Heart J. 2015;36(42):2921-64

5. Ferreira dos Santos L, Moreira D, Ribeiro P, Rodrigues B, Correia E, Nunes L, et al. Purulent pericarditis: A rare diagnosis. Rev Port Cardiol (English Ed). 2013;32(9):721-7.

6. Arsura EL, Kilgore WB, Strategos E. Purulent pericarditis misdiagnosed as septic shock. South Med J. 1999;92(3):285-8.

7. Lange RA, Hillis LD. Acute pericarditis. New Engl J Med. 2004;351(21):2195-202.

8. Vargas Cruz A, Gómez Álvarez E, Jaime Uribe A, Salgado Alday ME. Pericarditis purulenta: presentación de un caso y diagnósticos diferenciales. Arch Cardiol Mex. 2006;76(1):83-9.

9. Suberviola Cañas B, Rodríguez Borregan JC, González Castro A, Miñambres E, Burón Mediavilla FJ. Pericarditis purulenta y empiema pleural por Streptococcus pneumoniae. An Med Interna. 2007;24(1):35-7.

10. Augustin P, Desmard M, Mordant P, Lasocki S, Maury J-M, Heming N et al. Clinical review: Intrapericardial fibrinolysis in management of purulent pericarditis. Crit Care. 2011;15(2):220.

11. Vergara-López S, Pérez L, Corzo JE, Gómez-Mateos JM. Pericarditis purulenta como complicación de la neumonía neumocócica bacteriémica. Rev Clin Esp. 2008;208(10):531.

12. Burgess LJ. Biochemical analysis of pleural, peritoneal and pericardial effusions. Clin Chim Acta. 2004;343(1):61-84.

13. Martí V, Vila M, Ginel A, Domínguez de Rozas JM. Pericarditis purulenta complicada con taponamiento cardíaco debida a Streptococcus agalactiae. Rev Med Chile. 2010;138(4):519-20.

14. Pericarditis Empiric Therapy [Internet]. Medscape; 22 de septiembre de 2020 [citado: 1 de abril de 2021]. Disponible en: https://emedicine.medscape.com/article/1953143-overview 